\title{
BARRANCA DEL METLAC, VERACRUZ: UN PAISAJE PATRIMONIAL EN RIESGO
}

Muñoz-Márquez Trujillo, Rafael Arturo; Zárate-Rodríguez, Araís

BARRANCA DEL METLAC, VERACRUZ: UN PAISAJE PATRIMONIAL EN RIESGO

Revista Legado de Arquitectura y Diseño, vol. 15, núm. 27, 2020

Universidad Autónoma del Estado de México, México

Disponible en: http://www.redalyc.org/articulo.oa?id=477963263009

Esta obra está bajo una Licencia Creative Commons Atribución-NoComercial-SinDerivar 4.0 Internacional. 


\title{
BARRANCA DEL METLAC, VERACRUZ: UN PAISAJE PATRIMONIAL EN RIESGO
}

\author{
BARRANCA DEL METLAC, VERACRUZ: A \\ HERITAGE LANDSCAPE AT RISK
}

\author{
Rafael Arturo Muñoz-Márquez Trujillo arturom@colpos.mx \\ Colegio de Postgraduados, México \\ Araís Zárate-Rodríguez araiszarate@gmail.com \\ Colegio de Postgraduados, México
} Revista Legado de Arquitectura y Diseño,
vol. 15, núm. 27, 2020

Universidad Autónoma del Estado de México, México

Recepción: 15 Agosto 2019 Aprobación: 22 Noviembre 2019

Redalyc: http://www.redalyc.org/ articulo.oa?id=477963263009
Resumen: Los paisajes se pueden considerar como patrimonio, ya que contienen la herencia que la sociedad ha dejado a través de la forma en que gestiona su territorio, por medio de la relación de ésta con el medio que la rodea. El presente trabajo se desarrolla en la Barranca del Metlac, entre los municipios de Fortín e Ixtaczoquitlán, Veracruz, en la sección donde se trazó y construyó la antigua vía del ferrocarril "El Mexicano”. Dada la belleza del sitio y el legado de un tipo de edificios y construcciones, que precisan una etapa histórica en el desarrollo de México, este paisaje patrimonial fue registrado por pintores y fotógrafos en el siglo XIX, situación que respalda el carácter patrimonial del lugar. En la década de los 70, se construyeron nuevos puentes vehiculares y ferroviarios para cruzar la barranca, dejando la antigua vía en desuso, y afectando las visuales registradas por los artistas y fotógrafos. Además de ello, el desarrollo urbano también está teniendo impacto visual (entre otros). Este artículo presenta los resultados preliminares de un análisis del impacto de esos elementos, en el paisaje patrimonial, basado en el aspecto visual. Los resultados muestran que diversas visuales conspicuas son afectadas, tanto por las infraestructuras nuevas, como por el desarrollo urbano reciente.

Palabras clave: Cuenca visual, Veracruz, SIG.

Abstract: Landscapes can be considered as heritage, since they contain the heritage that society has left through the way it manages its territory, through its relationship with the surrounding environment. This work is carried out in the Barranca del Metlac, between the municipalities of Fortin and Ixtaczoquitlan, Veracruz, in the section where the old railroad track "El Mexicano" was drawn and built. Given the beauty of the site and the legacy of a type of buildings and constructions that require a historical stage in the development of Mexico, this heritage landscape was recorded by painters and photographers in the nineteenth century, a situation that supports the heritage of the place. In the 1970s, new vehicular and railway bridges were built to cross the ravine, leaving the old road in disuse, and affecting the visuals recorded by the artists. In addition to this, urban development is having visual impact as well (among others). This paper presents the preliminary results of an analysis of the impact of these elements, in the heritage landscape, based on the visual aspect. The results show that various conspicuous visuals are affected, both by new infrastructure and by recent urban development.

Keywords: Viewshed, Veracruz, GIS.

\section{INTRODUCCIÓN}

Los paisajes son un recurso de alta fragilidad (Muñoz-Pedreros, 2004), y son fuente de información que muestran la relación de la sociedad que los genera, con el medio natural y físico que los rodea, o como Besse lo indica, es un proyecto social, aun cuando la sociedad que los 
genera no esté consciente del mismo (Besse, 2006). Al paisaje lo han definido de diversas maneras, las cuales se han amoldado a la disciplina que lo cita Larrucea (2016), destacando diversos aspectos que, de ese concepto, le son útiles a cada una de ellas. En ese sentido, Besse (2006) identifica al menos cinco enfoques diferentes del concepto paisaje, expone cómo cada disciplina otorga un significado con rasgos y características diferentes entre sí. Es necesario destacar la conclusión que plantea dicho autor, cuando expresa que la falta de universalidad que debería poseer el concepto en sí mismo, demuestra que cada campo de aplicación lo emplea sin considerar referencias intelectuales similares. Ello nos coloca en una situación un tanto inestable desde el punto de vista conceptual, que requiere de rasgos y propiedades esenciales para delimitar adecuadamente el significado y fijar mejor una postura. Un punto de inflexión que se ha convertido en una referencia importante, en este sentido, es el "Convenio Europeo del Paisaje” (Consejo de Europa, 2000). En este documento, se entiende al paisaje, como "cualquier parte del territorio tal como la percibe la población, cuyo carácter sea el resultado de la acción y la interacción de factores naturales y/o humanos". Ahí se concibe una relación intrínseca y tal vez escalar, entre el territorio y el paisaje, dado que, esta definición indica, que un paisaje es alguna parte o sección de un territorio. Entonces, el territorio se establece como un marco físico (tangible), donde interactúan los fenómenos naturales y sociales, y a partir de la percepción (intangible) que los individuos tienen de alguna parte de él, es entonces que surge el paisaje. Pero ¿cómo se percibe el paisaje? La percepción sucede a partir de los sentidos, esto es la vista, el oído, el olfato, el gusto y el tacto, y, sin embargo, hay que considerar que estas percepciones son influenciadas por la cultura, por lo que la percepción del paisaje es de raíz cultural. En ese sentido, Maderuelos (2005) postula que "el paisaje es un constructo, una elaboración mental que los hombres realizamos a través de ciertos fenómenos de la cultura”.

Con respecto a lo patrimonial, se puede considerar el paisaje como el que concreta espacialmente la herencia recibida, y que además expresa la idiosincrasia cultural de un grupo social (Castillo, 2009). Patrimonio lo son las expresiones arquitectónicas, agrícolas o forestales, entre otras, y los aspectos del medio físico, que resultan de un determinado manejo de ese territorio, y que se expresa en un paisaje, el cual es patrimonio, simplemente por poseer dentro de él diversos elementos, tanto naturales como artificiales, que muestran esa relación de una determinada sociedad con su ambiente (Bolós, 1992). Los paisajes tienen que ver con las interacciones entre el sustrato físico-natural y la sociedad a través del tiempo; son un ente dinámico, cambiante y complejo. Transcurre su cambio a lo largo del tiempo y el espacio, es decir, en escalas temporales y espaciales. En este estudio, se analiza el cambio de un paisaje en el tiempo, compuesto por interesantes elementos naturales y antrópicos. Posee valores estéticos y ambientales importantes, que han sido reconocidos lustros atrás y registrado en el siglo XIX, por ejemplo, por pintores y fotógrafos, con lo que se posee un registro visual del estado pasado y presente en ese espacio. Se trabaja con una sección de la Barranca de 
San Miguel, barrera física natural entre los municipios de Fortín de las Flores e Ixtaczoquitlán, en el estado de Veracruz, México. Se analizan los impactos en lo visual, por modificaciones antrópicas, por medio del uso de tecnologías de Sistemas de Información Geográficos (SIG). Se presentan resultados preliminares de este análisis y se generan recomendaciones para considerar en aspectos de gestión del paisaje en el desarrollo urbano y regional, con miras a la preservación paisajística y ambiental, elementos necesarios para la sociedad.

\section{METODOLOGÍA}

El objetivo general de esta investigación fue determinar las afectaciones en las visuales más conspicuas en la Barranca de San Miguel, entre los municipios de Fortín e Ixtaczoquitlán, Veracruz, conocido también como Barranca de Metlac, paisaje patrimonial, en relación al crecimiento urbano y otros procesos antrópicos, de los últimos 50 años. Como objetivo se determinó, analizar el impacto visual del desarrollo urbano e infraestructura, en los puntos más notables de la barranca, a partir de estudios de cuencas visuales, con base en el recorrido del segmento de la línea ferroviaria que perteneció al Ferrocarril el Mexicano, y determinar el grado de impacto de dichos cambios en el paisaje analizado.

El área de estudio, como se ha comentado, corresponde a la Barranca del Metlac (Barranca de San Miguel), que se localiza en el municipio de Fortín de las Flores e Ixtaczoquitlán, Veracruz, entre las coordenadas UTM 2090000 y 2093000 N, y 7070000 y 712000 E y a una altitud promedio de 950 m.s.n.m. (figura 1).

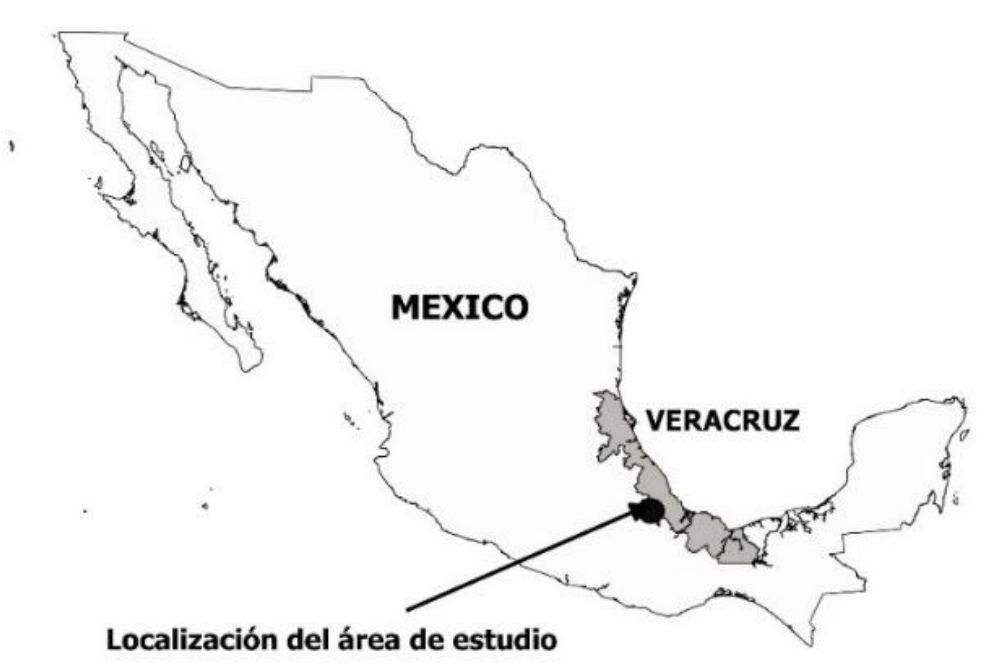

Figura 1. Localización del Área de Estudio. Fuente: Elaboración propia.

La zona seleccionada para la realización de este estudio, se muestra en la figura 2, como se observa, el área de trabajo comprende de los $\mathrm{km} 303$ al 
309 de la antigua línea de tren del "Ferrocarril el Mexicano", y alrededor de $3 \mathrm{~km}$ al norte y $3 \mathrm{~km}$ al sur, considerando el nuevo puente de Metlac, de nombre oficial, "Ingeniero Mariano García Sela", los mismo al este y al oeste del mismo punto, resultando en alrededor de $36 \mathrm{~km}^{2}$ de área de trabajo (figura 2).

Este espacio posee una serie de elementos de importancia constructiva y ambiental. Con respecto de los primeros, el cuadro 1 muestra un recuento de varios de ellos. En este trabajo no se desarrolla una descripción amplia de los edificios y la obra civil patrimonial, pero una exploración más profunda y descriptiva se puede encontrar en Zárate (2011). Acerca de esas edificaciones, es importante mencionar que se construyeron para la operación del Ferrocarril el Mexicano, y que varias de ellas se encuentran con diferentes grados de alteración y deterioro. Se trata de un conjunto de edificios arquitectónicos e ingenieriles, los cuales son evidencia física y testimonio histórico, entre otras cosas, del intercambio de conocimiento de diferentes culturas para solucionar las condiciones geográficas de un territorio con barreras naturales de extraordinaria belleza y complejidad. Importante puntualizar que el caso específico de la Barranca de Metlac se constituyó entre los retos más difíciles de solucionar técnicamente, en la introducción del sistema de ferrocarril en México (Zárate, 2011). 


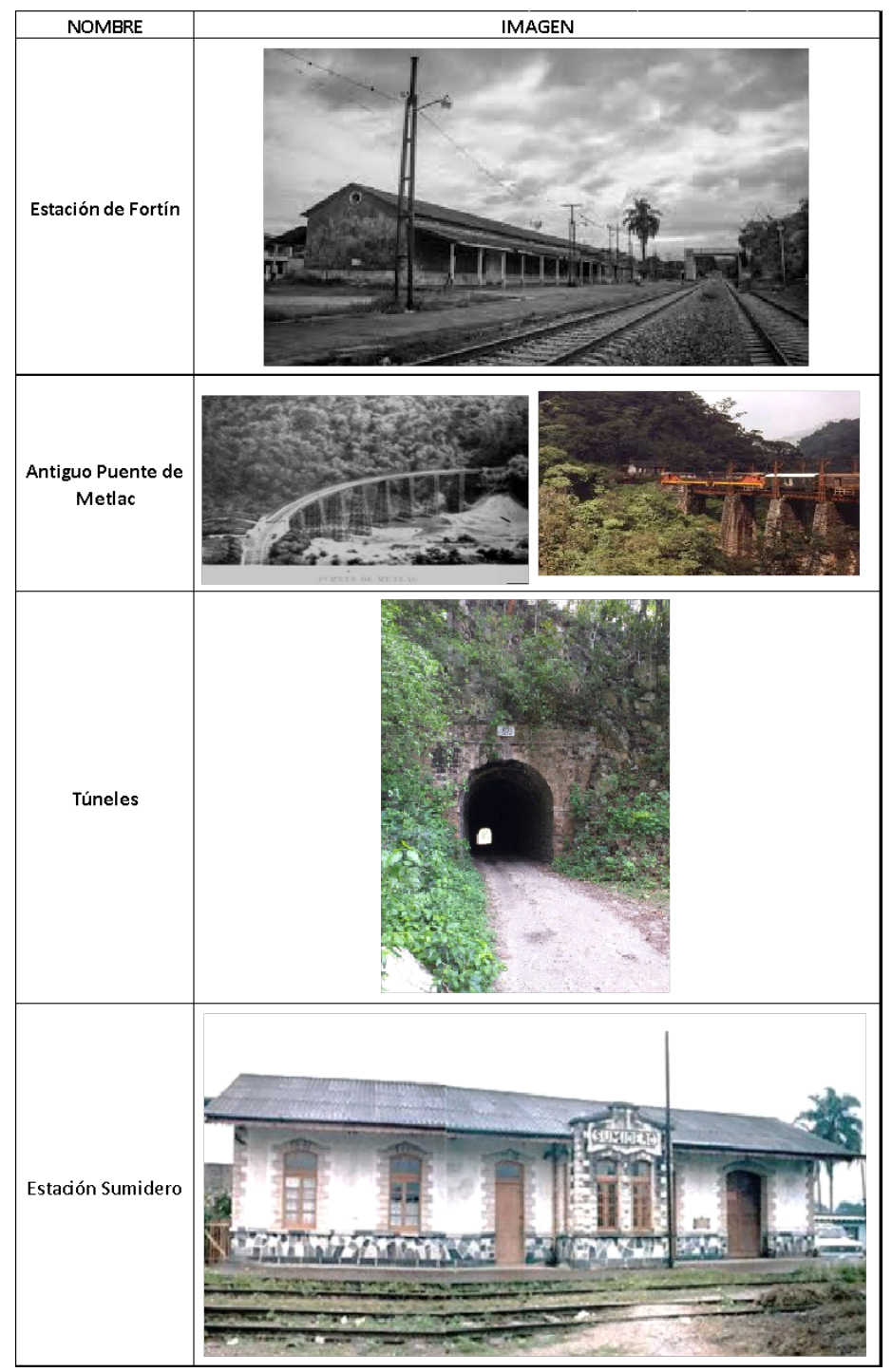

Cuadro 1. Algunas edificaciones de importancia en el área de estudio. Fuente: Elaboración propia con base en Zárate (2011). 


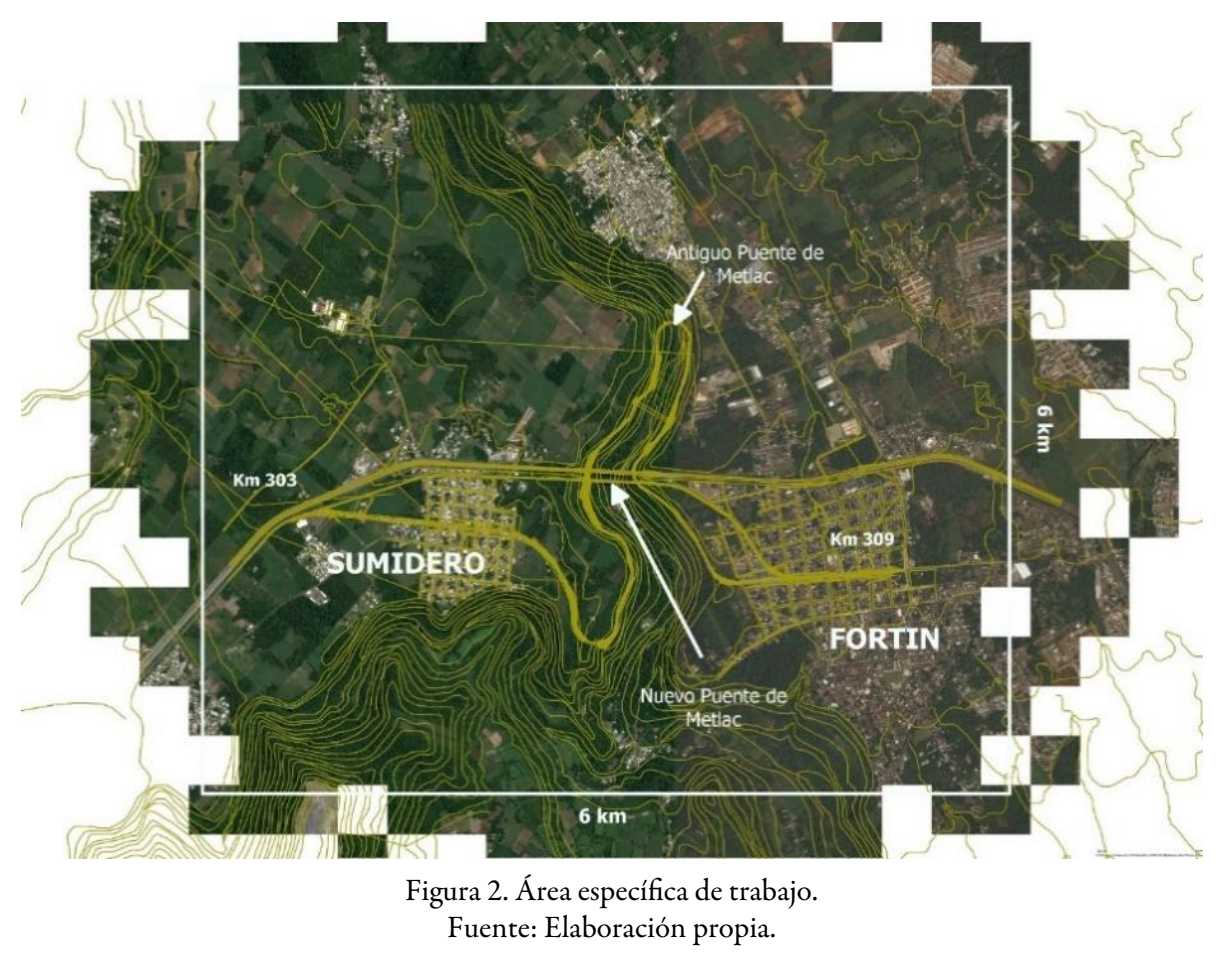

En cuanto a su riqueza biológica, la vegetación está constituida por bosque mesófilo de montaña, bosque de pino-encino y fragmentos de selva alta y mediana subperennifolia y selva baja caducifolia. Algunas de las especies representativas del lugar son el liquidámbar (Liquidámbar styraciflua), marangola (Clethra mexicana), pipinque (Carpinus caroliniana), haya (Platanus mexicana), pino (Pinus pseudostrobus), encinos (Quercus oleoide y Q. macrophylla), palo mulato (Bursera simaruba), roble rosa (Tabebuia rosea), vainillo (Inga vera) y fresno (Fraxinus uhdei). La fauna se encuentra representada por diversas especies de anfibios, reptiles, aves y mamíferos. Es el único paso libre entre la zona del Pico de Orizaba y los bosques mesófilos y bosques tropicales de Huatusco y Coscomatepec hacia la región de Zongolica y Tezonapa, puesto que la autopista Córdoba-México representa una barrera importante para el flujo de la vida silvestre del centro de Veracruz, dicha barranca permite que se mantenga la conectividad ecológica entre estas dos grandes zonas de Veracruz. Por todo esto, se considera que esta zona tiene una gran prioridad de conservación (Rivera, 2015).

En cuanto a la memoria histórica, es importante comentar que, dada su belleza, este sitio fue pintado por artistas como José María Velasco y Casimiro Castro, alrededor de los años 1890 (figura 3).

Como se ha descrito se trata de un espacio de características excepcionales con respecto a los bienes patrimoniales bioculturales, y paisajísticos que alberga. Este lugar ha sido afectado, desde la construcción de la primera vía del tren fechada entre 1872 y 1873 (Zárate, 2011), y a través de los años, a causa de la construcción de infraestructura carretera y el desarrollo urbano de las localidades vecinas, entre otras causas. Con la intención de conocer y realizar una evaluación de los impactos de esas estructuras en lo visual, en este trabajo, se desarrolló una evaluación 
cualitativa de ellas a partir del eje de la antigua vía del Metlac, en el tramo descrito. Se realizó un análisis con la utilización de un SIG, investigación documental, y verificación de datos en campo. Para determinarlos, se presentan en este artículo, los aspectos más relevantes obtenidos de este estudio.

El trabajo se desarrolló en dos fases: a) investigación bibliográfica, cartográfica y de campo, y b) desarrollo de un estudio de cuencas visuales, por medio de la utilización del SIG, y otros datos de elevación, así como imágenes de satélite.

Para determinar impactos en las visuales por edificaciones o incremento de la mancha urbana, se utilizó QGIS 2.18.21, así como Global Mapper. Se contó con datos de relieve del Instituto Nacional de Estadística, Geografía e Informática Lidar Superficie, esc. 1:10,000, resolución 15 m (INEGI, 2017), a fin de realizar un Modelo Digital de Elevación (MDE). De esa manera se desarrolló una capa ráster, con pixeles de 15 x 15 m cada uno. Se contó con un archivo Autocad de levantamiento de la vía, con cadenamientos a cada $20 \mathrm{~m}$, previamente realizado, además de varias visitas de campo de verificación de datos y resultados. Una vez desarrollado el MDE, se construyó una capa vectorial de puntos, que se distribuyeron a cada $100 \mathrm{~m}$, sobre el eje central de la antigua vía férrea, generándose 75 puntos de observación (figura 4).

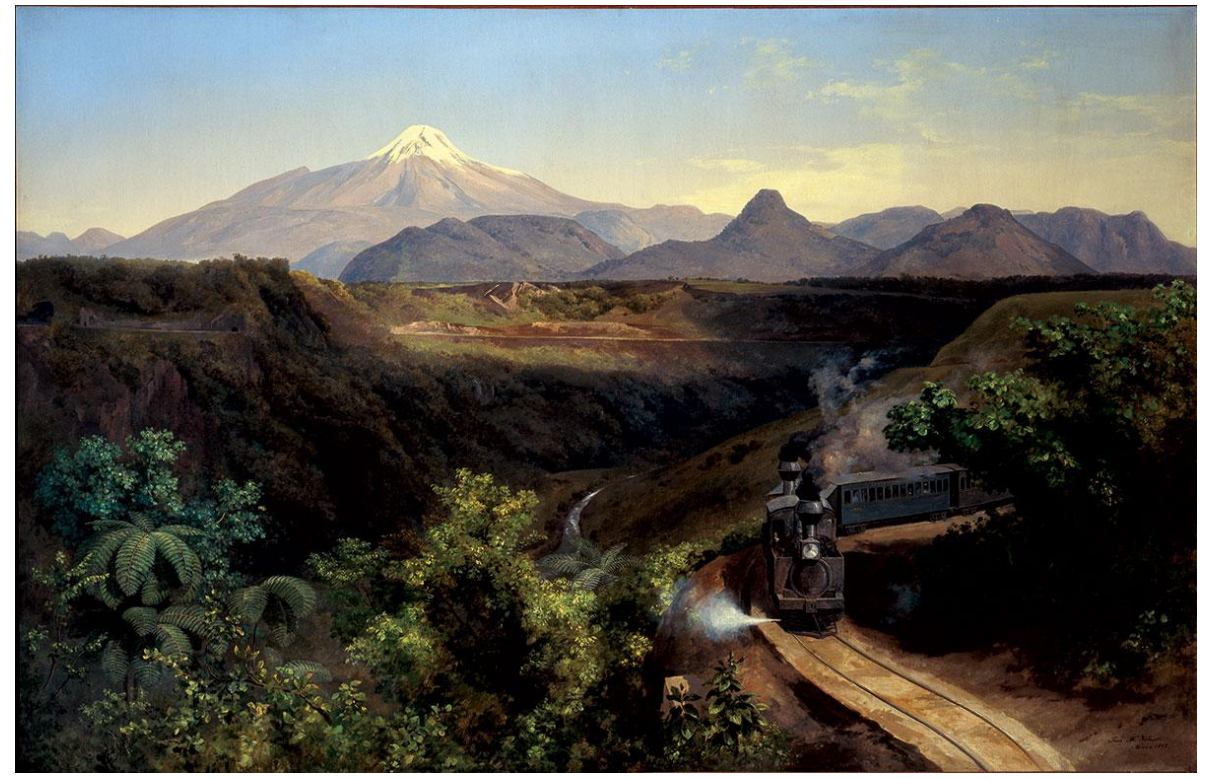

Figura 3. "Cañada de Metlac".

Fuente: Velasco, J. M. (1897). 


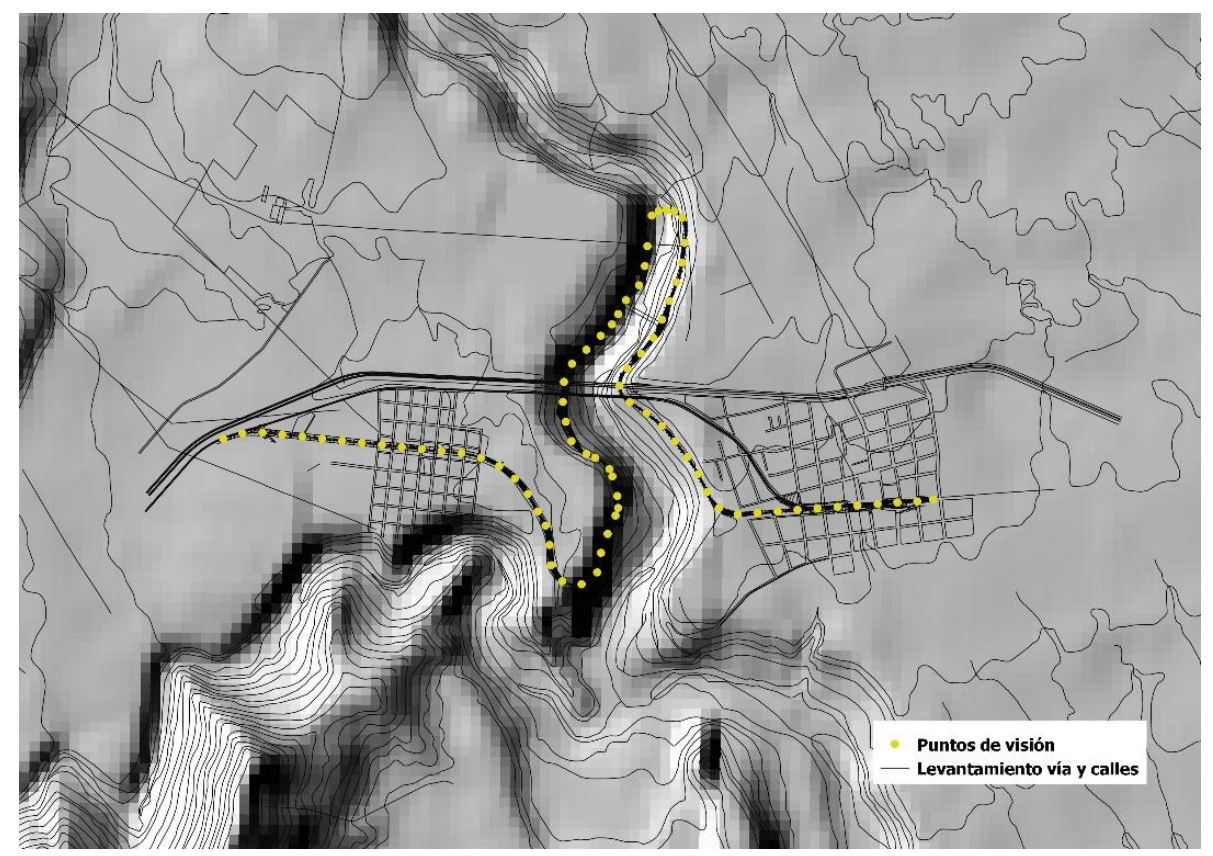

Figura 4. Puntos de observación colocados en la vía. Fuente: Elaboración propia.

Utilizando la rutina "Viewshed" (cuenca visual) en QGIS; y con el MDE y la capa vectorial de puntos de observación, se generaron cuencas visuales con valores acumulados, es decir, la generación de una capa que reflejara los valores que cada pixel es visto desde algún punto a lo largo de la vía: los valores más altos indican que ese o esos pixeles son vistos por más puntos, mientras que los que tienen valores bajos, o iguales a cero, quiere decir, que se trata de un pixel que es visto por pocos puntos, o por ninguno.

\section{RESULTADOS Y DISCUSIÓN}

Dentro de los impactos encontrados, al paisaje patrimonial en el sitio, se pueden enumerar dos principales:

1. Construcción del puente Ingeniero Mariano García Sela, y el puente nuevo del ferrocarril, ambos en la década de los años 70 . Con respecto al primero, se trata de una estructura de $368 \mathrm{~m}$ de largo, que se levantó a una altura de $110 \mathrm{~m}$ desde la parte baja de la barranca, donde discurre el Río Metlac (Ramírez, 2018). Sin duda un ejemplo de la gran ingeniería civil mexicana, pero que desde el punto de vista de impacto visual (y ambiental), invadió el espacio inmortalizado por el artista, José María Velasco, expuesto por Fausto Ramírez, historiador de arte, como el referente del paisajismo decimonónico mexicano, en su libro José María Velasco, pintor de paisajes (Ramírez, 2017). En la figura 5 se muestra el punto donde se determinó que el artista colocó el caballete para realizar el óleo que se muestra en la figura 3. Como se puede apreciar, en la figura 6, la visual se bloquea con el puente vehicular actual. Esto, definitivamente, retira la posibilidad de poder apreciar el paisaje, desde dónde y cómo lo observó el artista. Este es un gran impacto en la visual patrimonial 
plasmada en la pintura de paisaje de la barranca, donde la composición de los elementos naturales de la Barranca, y el frente de la locomotora del tren El Mexicano se acompañan por un fondo majestuoso por la presencia del Citlaltépetl o también llamado Pico de Orizaba, volcán con la elevación más alta en el país.

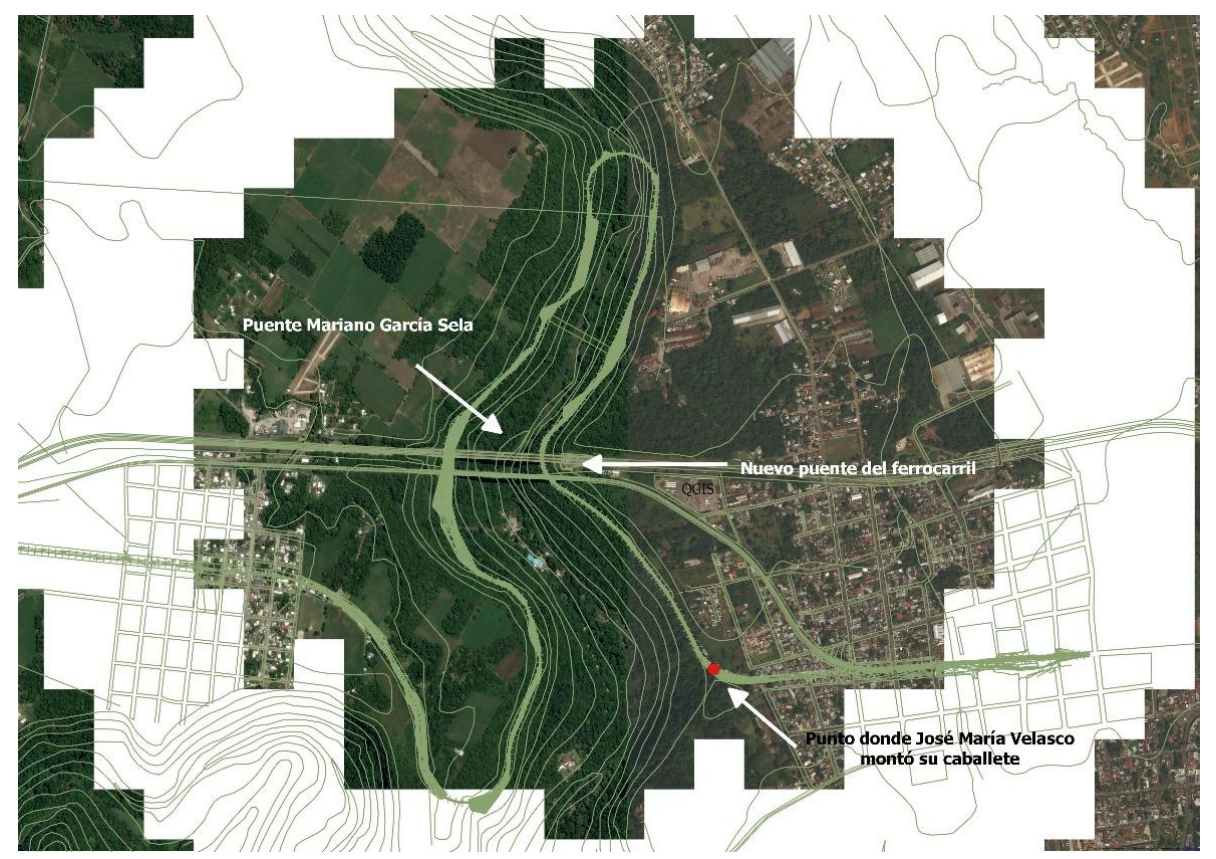

Figura 5. Ubicación del punto donde se estima, José María Velasco realizó la obra "La Cañada de Metlac". Fuente: Elaboración propia.

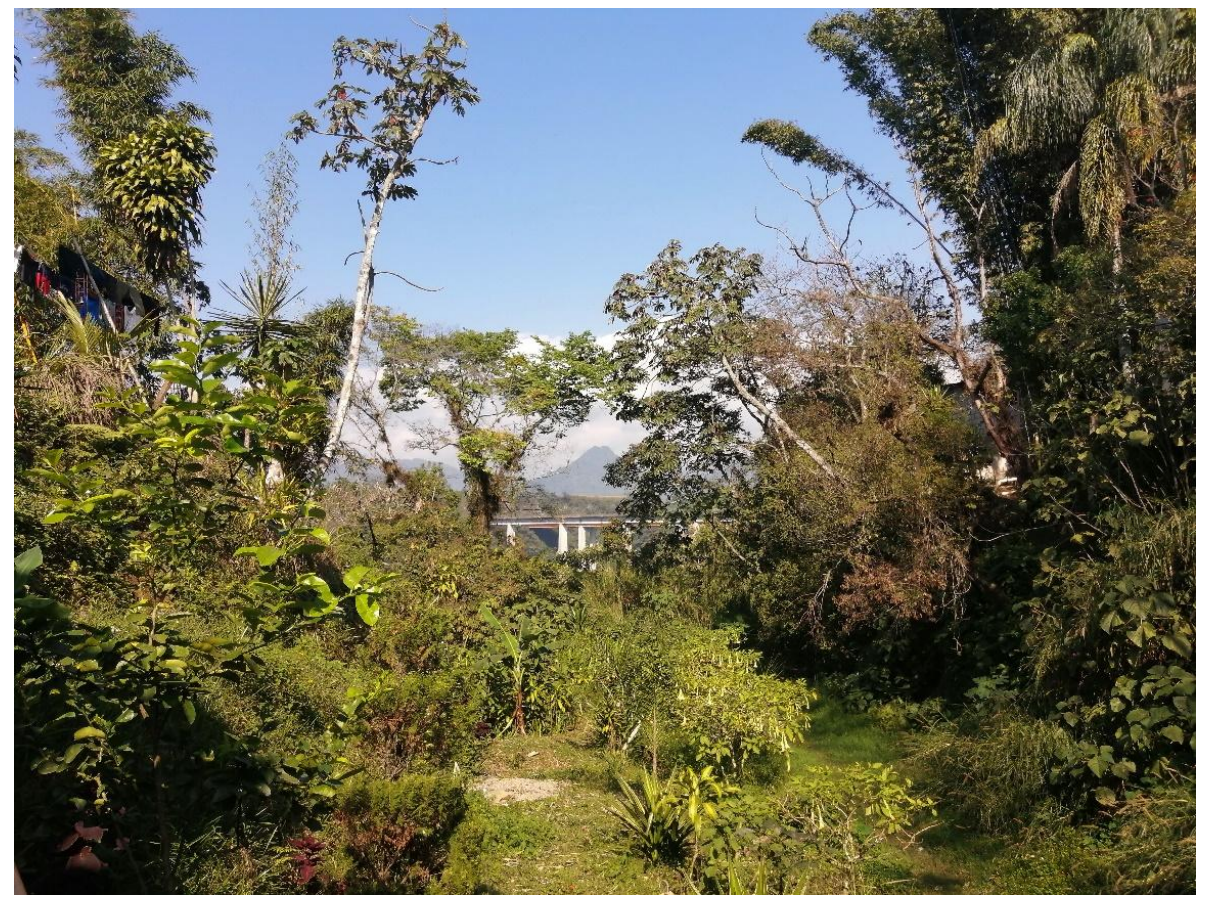

Figura 6. Impacto visual (y ambiental) con referencia al paisaje pintado por José María Velasco en su obra "Cañada de Metlac” (1893). Fuente: Elaboración propia.

2. Impacto en las visuales a partir de puntos de observación de la antigua vía del ferrocarril, a causa de las nuevas estructuras y el desarrollo 
urbano. Como se explicó en el apartado de metodología, a través de un estudio de cuencas visuales, se generaron mapas binarios a partir de cada punto. Estos mapas indican, qué pixel se ve y cuál no se ve, a partir de un punto determinado. Aplicando álgebra de mapas, y sumando todos los mapas resultantes a partir de cada punto de la vía (en este caso, 75), se llegó a un mapa-resultado, que muestra qué áreas del contexto de la vía, son más visibles que otras, a partir de todos los puntos. Sobreponiendo ese mapa resultante, en una imagen de satélite del territorio, se pudo corroborar la presencia de elementos que impactan las vistas, sobre todo por aspectos como el desarrollo urbano. De esa manera, la figura 6 muestra ese resultado. Dicha imagen indica los pixeles que son visibles por la suma de puntos que se encuentra en el cuartil más alto de valores obtenidos. En el cuadro 2 se observan algunos ejemplos de los impactos obtenidos por este método y calibrados en campo (mayormente impactos por el reciente desarrollo urbano).

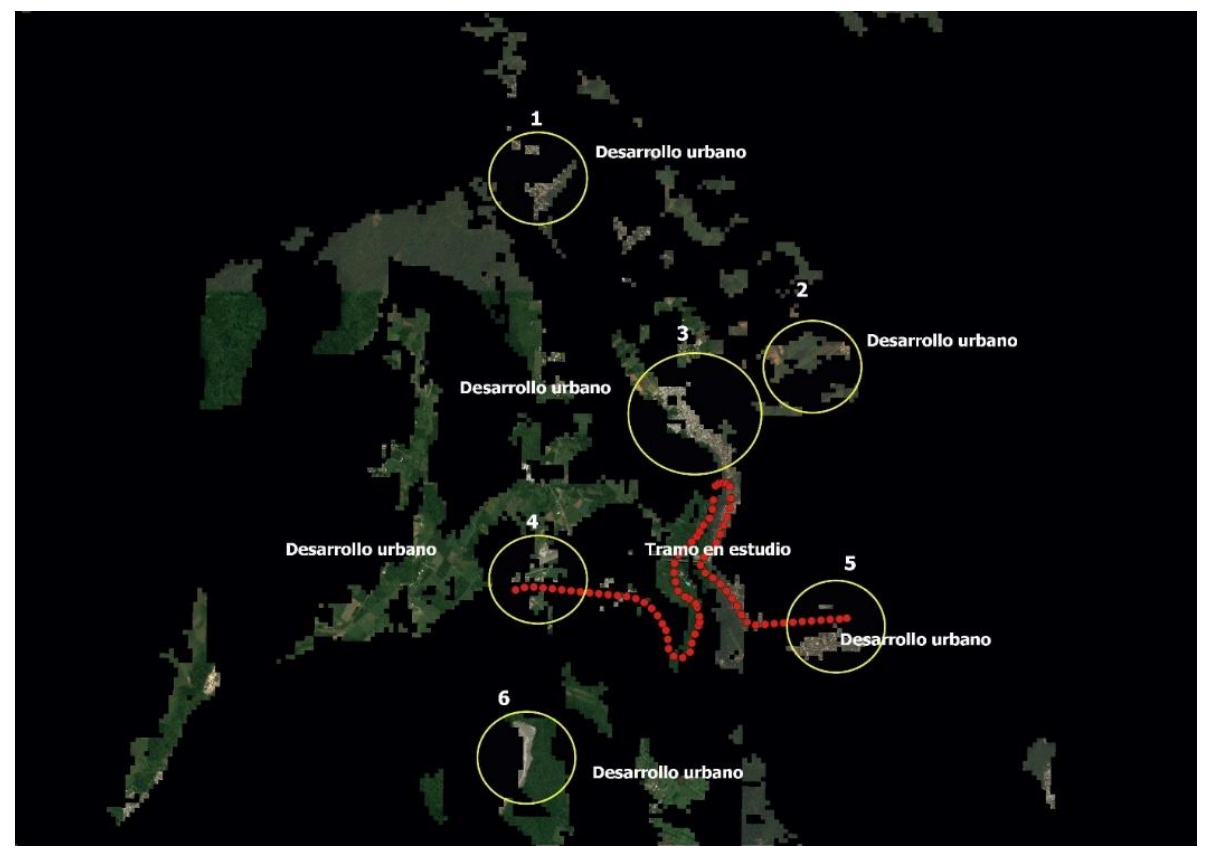

Figura 7. Puntos conflictivos por el desarrollo urbano, en relación con las visuales desde la vía. Las zonas en negro no se visualizan desde la vía. Fuente: Elaboración propia.

Estos resultados exploran algunas de las variables que han venido afectando al sitio patrimonial desde el punto de vista solamente visual. No se mencionan otros aspectos que están siendo presentes en el lugar, por ejemplo, el cambio de uso de suelo, o la contaminación, y el impacto de acciones de deforestación, el vandalismo y la alteración en las edificaciones históricas, también el robo de las vías y durmientes de la línea del tren, así como puntos de descargas de aguas negras de las colonias en la parte superior de la barranca, las cuales se tiran directamente por los taludes, generando inestabilidad en la superficie y generando deslizamiento de laderas, por mencionar algunos más. Sin embargo, los resultados documentan cómo el impacto, en el día de hoy a la barranca, es importante. Poco queda de aquello que los paisajistas y fotógrafos del siglo XIX registraron en su pintura. Sirva este trabajo para dejar 
de manifiesto la necesidad de gestionar adecuadamente el manejo de espacios patrimoniales como lo es esta barranca, donde es imprescindible el tema de conservación por ser el único corredor biológico en la región, la preservación por contener parte del legado histórico arquitectónico, e industrial ferroviario de nuestro país, aspectos que influyeron en las dinámicas territoriales y formando parte de los bienes culturales de las localidades que se desarrollaron directa e indirectamente de la actividad del ferrocarril, y sobre todo para tener una base para planificar y manejar ese espacio que brinde un beneficio social y ambiental. Evidentemente no se podrá demoler los nuevos puentes, por ejemplo, pero sí se debe gestionar el manejo y preservación de lo que queda, en un estado aceptable.

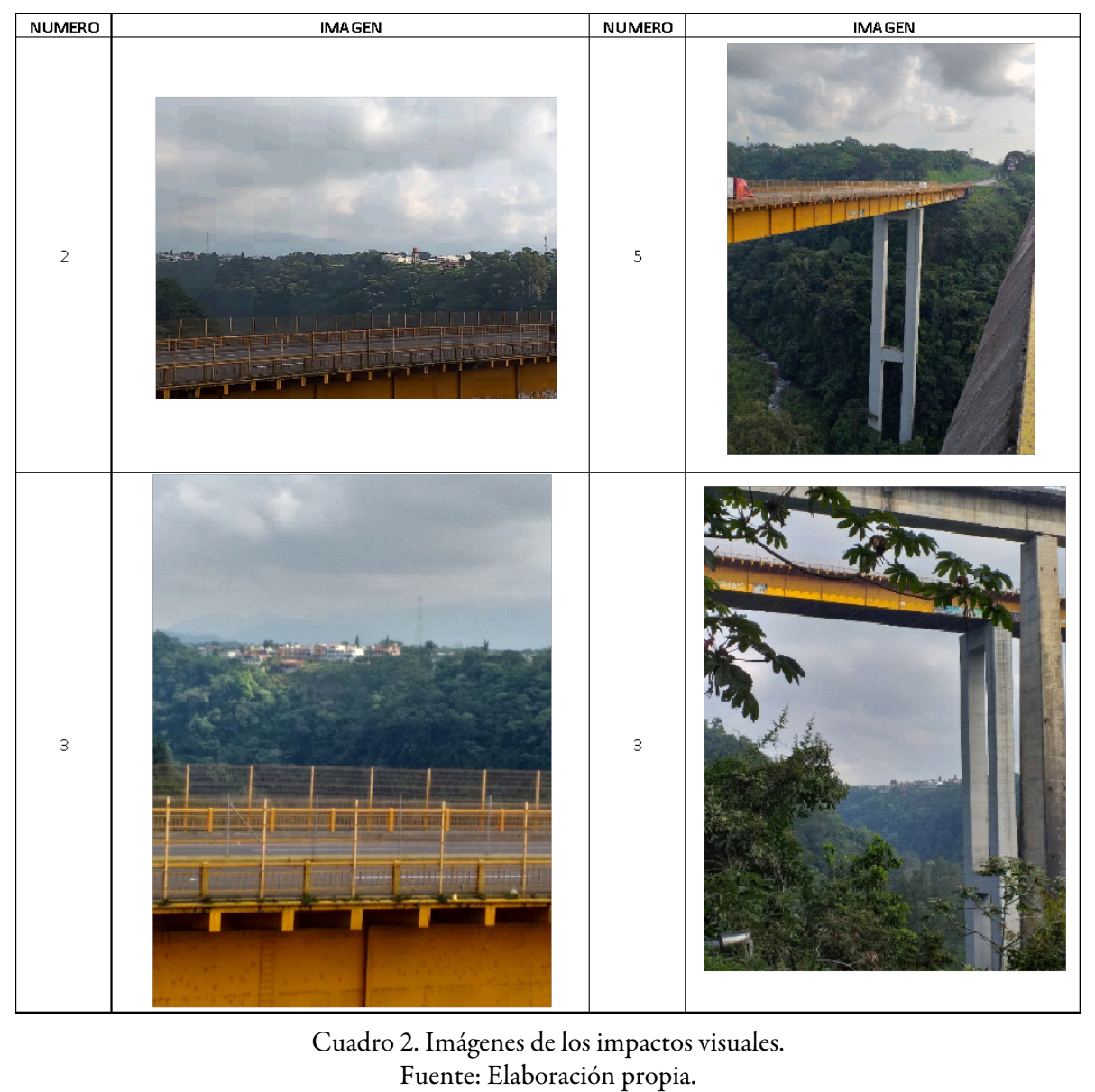

CONCLUSIONES

El trabajo desarrollado, arroja información importante que debe ser considerada para las políticas de manejo de este paisaje patrimonial, dados los impactos detectados desde lo visual. Por lo anterior, se considera que el objetivo de desarrollar un análisis cualitativo de los impactos al patrimonio, ha sido satisfecho adecuadamente, y pueda ser tomado como una base para generar propuestas de uso del sitio que sean respetuosas de este espacio, a través de acciones como la generación de un plan maestro de la zona que contemple, entre otros aspectos, el impacto 
visual de estructuras nuevas y del desarrollo urbano aledaño. Como recomendación se plantea la necesidad de realizar análisis cuantitativos de impactos en el paisaje, que complementen los resultados, para con ello generar políticas públicas que se destinen a subsanar deterioros.

Desde el punto de vista metodológico, la secuencia de pasos seguidos, permitió arribar a resultados que se corroboraron en campo, lo cual indica que se trata de una herramienta adecuada para aspectos exploratorios de impacto visual a paisajes patrimoniales, y sobre todo replicarse a otras regiones o espacios, inclusive aguas arriba de la zona analizada.

\section{FUENTES DE CONSULTA}

Besse, J. M. (2006), "Las cinco puertas del paisaje. Ensayo de una cartografía de las problemáticas paisajeras contemporáneas”. En Marchán, F. S. y Maderuelos, J. Paisaje y pensamiento, Abada Editores, Madrid, pp. 145-172.

Bolós, I. C. (1992), Manual de ciencia del paisaje: teoría, métodos, Masson, Barcelona.

Castillo, R. J. (2009), "La dimensión territorial del patrimonio histórico". En Patrimonio histórico y desarrollo territorial, Universidad Internacional de Andalucía, España, pp. 26-71.

Consejo de Europa (2000), Convenio Europeo de Paisaje. Disponible en http:// www.upv.es/contenidos/CAMUNISO/info/U0670786.pdf, consultado el 30 de septiembre de 2017.

Instituto Nacional de Estadística y Geografía (INEGI) (2017), Productos. Disponible en http://www3.inegi.org.mx/sistemas/biblioteca/ficha.aspx? upc $=702825004441$, consultado el 11 de enero de 2017.

Larrucea, G. A. (2016), País y paisaje: dos invenciones del siglo XIX mexicano, Universidad Nacional Autónoma de México, Facultad de Arquitectura, México.

Maderuelos, J. (2005), El paisaje: génesis de un concepto, Abada Editores, Madrid.

Muñoz-Pedreros, A. (2004), "La evaluación del paisaje: una herramienta de gestión ambiental”, Revista Chilena de Historia Natural, Issue 77, pp. 139-156.

Ramírez, A. (2018), Ingenieros Civiles y Puentes, Universidad Autónoma del Estado de México, México.

Ramírez, F. (2017), José María Velasco, pintor de paisajes, Universidad Nacional Autónoma de México, Instituto de Investigaciones Estéticas, Fondo de Cultura Económica, México.

Rivera, H. J. E. (2015), Flora, vegetación y priorización de áreas de conservación del Parque Nacional Cañón del Río Blanco, Veracruz, México, Instituto Tecnológico de Costa Rica, Costa Rica.

Velasco, J. M. (1897), "La cañada de Metlac" [Óleo sobre tela]. Museo Nacional de Arte. México. Disponible en http://munal.emuseum.com/objects/580/la-canada-demetlac?ctx $=13 \mathrm{db} 5 \mathrm{f} 61-62 \mathrm{~d} 3-4 \mathrm{aea}-\mathrm{b} 17 \mathrm{a}-\mathrm{b} 80439 \mathrm{e} 0916 \mathrm{f} \& \mathrm{didx}=0$, consultado el 10 de agosto de 2019. 
Zárate, R. A. (2011), Análisis del valor arquitectónico y parámetros de reutilización de los edificios del Ferrocarril Mexicano en la Barranca de Metlac. Tesina de Arquitectura, Universidad Veracruzana, Facultad de Arquitectura, Córdoba. 